\title{
Complementing X-ray \& Neutron Diffuse Scatter Analysis with STEM to Understand Relaxor Behavior
}

Abinash Kumar ${ }^{1}$, Jonathon Baker ${ }^{2}$, Matthew Cabral $^{3}$, Preston Bowes ${ }^{2}$, Shujun Zhang ${ }^{4}$, Elizabeth Dickey $^{2}$, Douglas Irving ${ }^{2}$ and James LeBeau ${ }^{1}$

${ }^{1}$ Massachusetts Institute of Technology, Cambridge, Massachusetts, United States, ${ }^{2}$ North Carolina State University, Raleigh, North Carolina, United States, ${ }^{3}$ University of Sydney, Sydney, New South Wales, Australia, ${ }^{4}$ University of Wollongong, Wollongong, New South Wales, Australia

Correlating the local structure of relaxor materials with their electromechanical behavior remains a major challenge. In the prototypical relaxor-piezoelectric system, $\mathrm{Pb}\left(\mathrm{Mg}_{1 / 3} \mathrm{Nb}_{2 / 3}\right) \mathrm{O}_{3}-\mathrm{XPbTiO}_{3}(\mathrm{PMN} \mathrm{PT})$, X-ray and neutron scattering experiments have been predominantly performed to resolve the structural variation. The similar features in the scattering data can, however, be explained by two contrasting theories. One predicts the presence of polar nanoregions in a non-polar matrix while other predicts a slush-like response in a continuum of multi-domain states [1,2]. Furthermore, recently observed differences in X-ray and neutron scattering have pointed to the importance of accounting for anions displacements in conjunction with the cations. While significant progress has been made with diffraction alone, high-resolution STEM imaging offers the ability to directly capture the local structure in PMN-PT, including the cations and anions. In this presentation, we will show how combining X-ray \& neutron diffuse scattering results with STEM analysis can reveal the structural variation in PMN-PT. We will demonstrate that annular dark field (ADF) and integrated differential phase contrast (iDPC) STEM imaging can simultaneously provide the locations of all cations ( $\mathrm{Pb}, \mathrm{Mg}, \mathrm{Nb} \& \mathrm{Ti}$ ) and anions (O) positions for different composition in PMN-PT system and also reveal chemical ordering, Figure 1. Krogstadet. al observes various contributions (C1-C4) in scattering data which predict local structure in PMN-PT [3]. Here, we describe these scattering features in terms of STEM analysis results. Diffuse scattering contribution $\mathrm{C} 1$, shows symmetric features in X-ray while in neutron scattering it become asymmetric. This reveals importance of correlated oxygen atom displacement to determine local structure. The $\mathrm{C} 1$ diffuse scatter contribution increases with PT content and the oxygen distance map for $\langle 110>$ direction in iDPC STEM image show strip like features which correlate with monoclinic like distortions, Figure 2 a\&b. We will show how these distortions also increase with PT content. Similarly, we will discuss how C2 \& C3 features can be correlated with chemically ordered regions (Figure 1b), while C4 can be explained by variation in $\mathrm{Pb}$ displacements due to presence of different cations, Figure $2 \mathrm{c}$. Finally, we will highlight the new insights that can be drawn by complementing results from diffuse scattering with atomic resolution STEM [4]. 
(a)
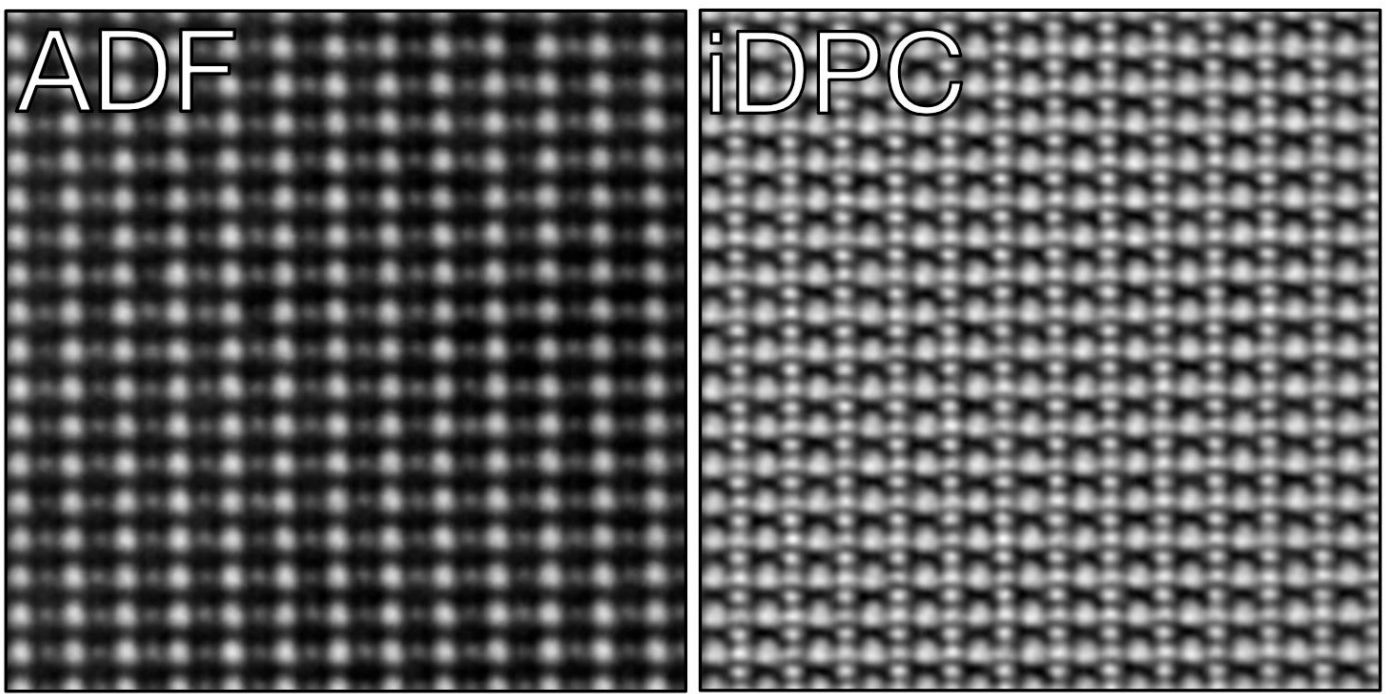

(b)
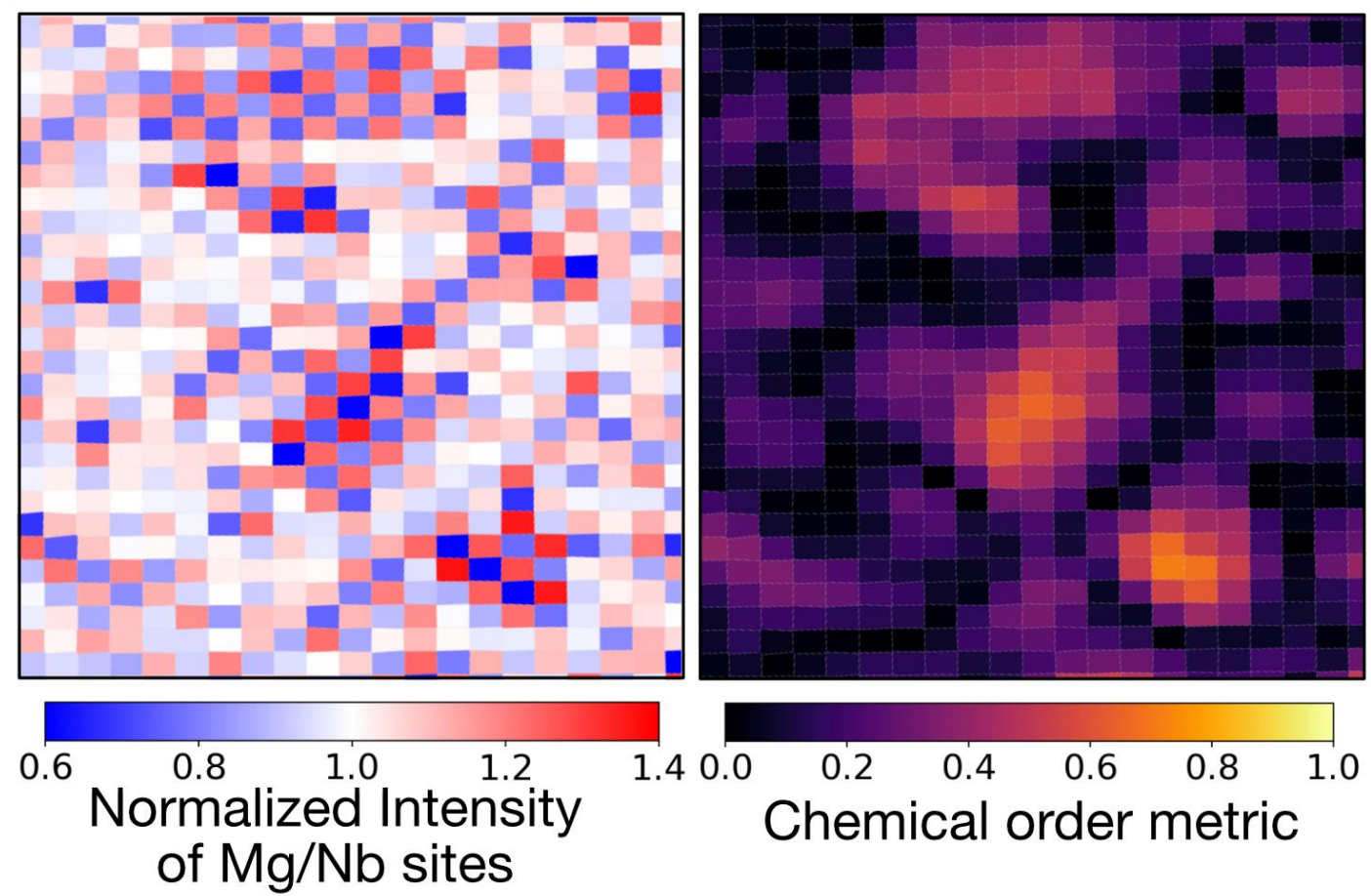
Chemical order metric

Figure 1. (a) ADF \& iDPC image of PMN along [110] (b) normalized intensity of $\mathrm{Mg} / \mathrm{Nb}$ sites, revealing chemical order regions in PMN 
(a)

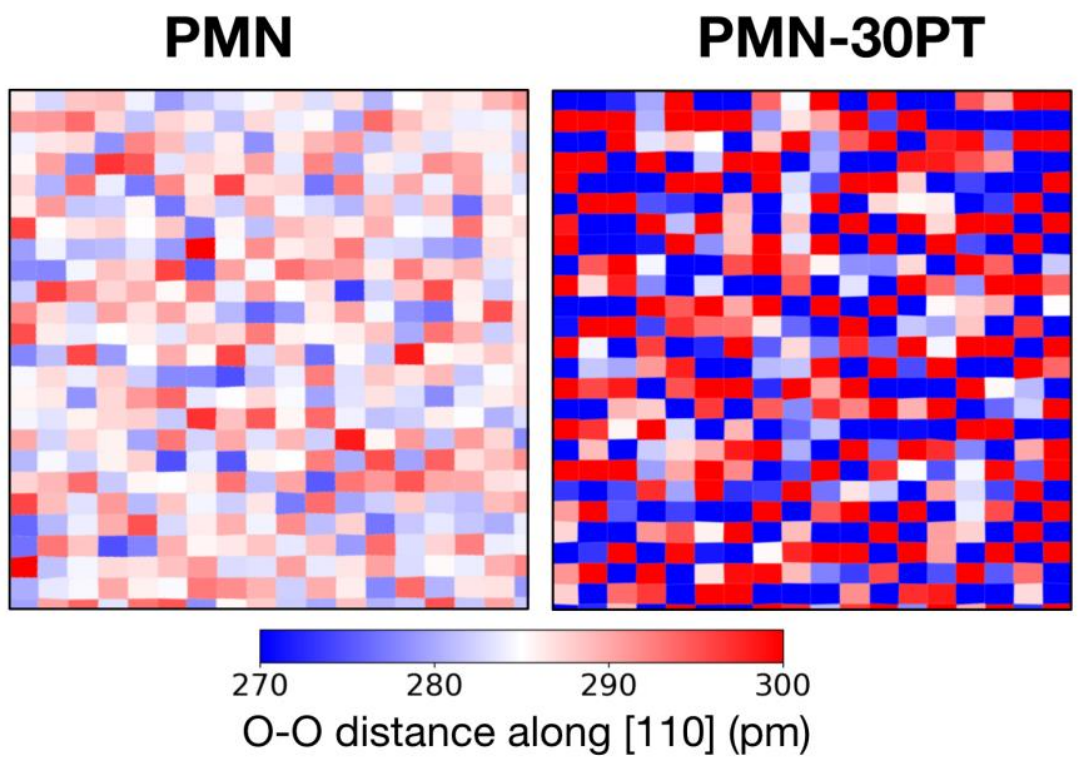

(b)

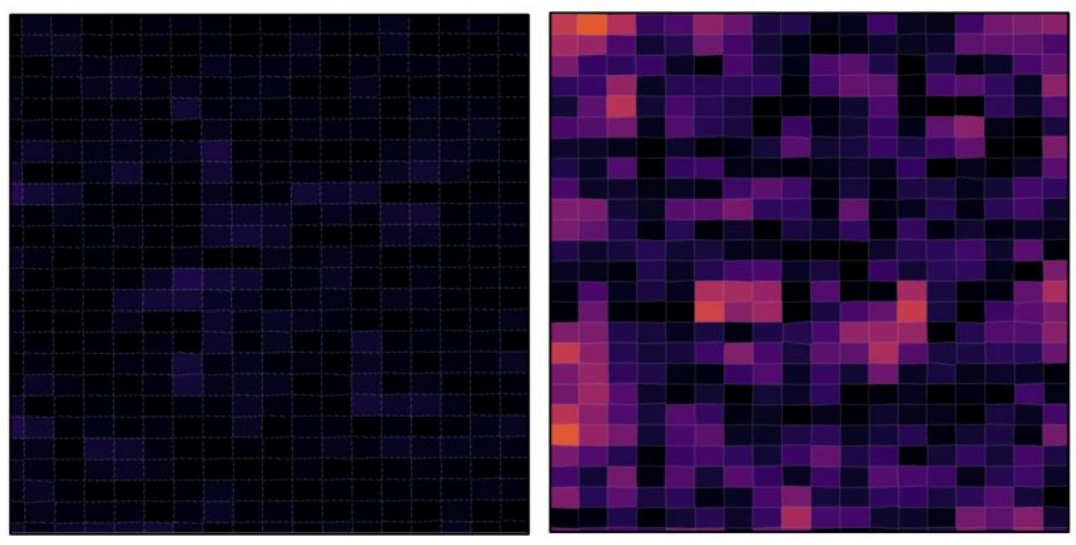

Monoclinic like distortion correlation

(c)

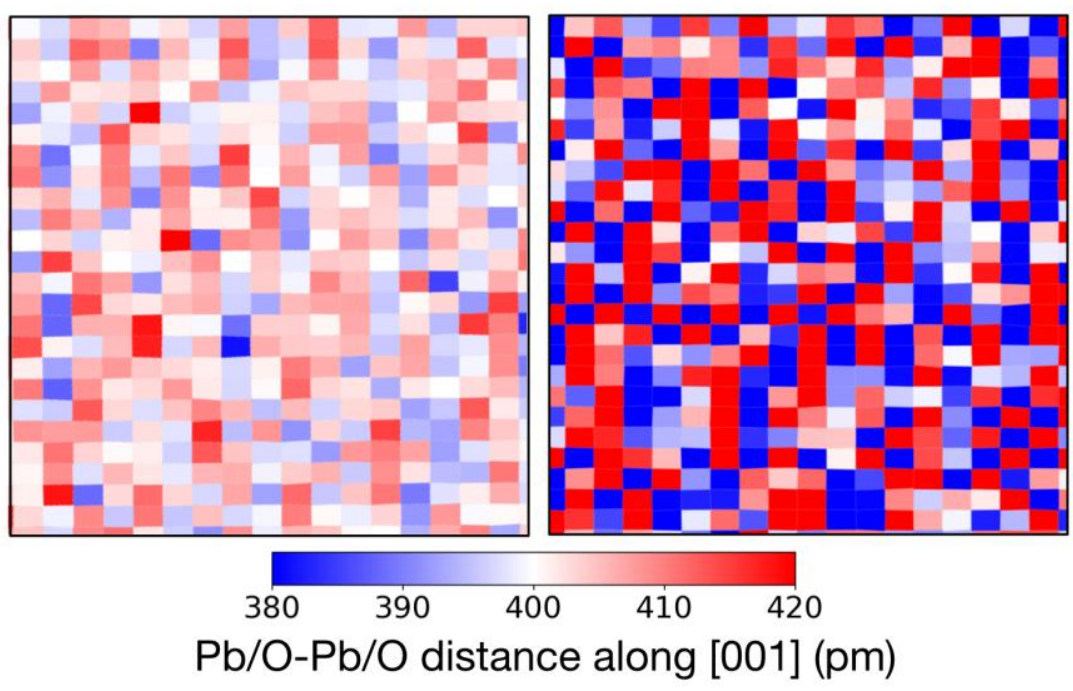

Figure 2. (a) Oxygen-Oxygen distance map along [110] (b) correlated monoclinic like distortion in octahedra \& (c) $\mathrm{Pb} / \mathrm{O}-\mathrm{Pb} / \mathrm{O}$ distance map along [001] in PMN \& PMN-30PT. 


\section{References}

[1] H. Takenaka, I. Grinberg, S. Liu and A. M. Rappe, Nature 546 (2017), p.391.

[2] F. Li et al., Nature Communications 7(2016), p. 13807.

[3] M. J. Krogstad et al., Nature Materials 17(2018) p. 718-724.

[4] This material is based upon work supported by the National Science Foundation, as part of the Center for Dielectrics and Piezoelectrics under Grant Nos. IIP-1841453 and IIP-1841466. 